\title{
EL DERECHO Y LA FUERZA
}

Hay más violencia en un jardín inglés que en la peor de las calles de una gran ciudad.

(P. D. James, Intrigas y deseos)

¿Ha habido alguna época de la Historia en que las gentes no se hayan sentido desdichadas? En verdad, todas las épocas se han considerado sin ventura, náufragas y perdidas... La nuestra no podría escapar a ese apocalíptico destino.

(Jorge Millas, Anuario de Filosofía Jurídica y Social, $\mathrm{N}^{\mathrm{o}}$. 2, Valparaíso, 1984)

\section{I}

\section{Sobre Elías Díaz y «Doxa»}

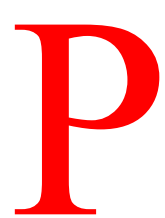

ocas cosas pueden ser más gratas para mí que colaborar en un homenaje a Elías Díaz. Después de hacer mi doctorado en la Universidad Complutense de Madrid en los inciertos años españoles de 1975 y 1976, tuve luego oportunidad de volver a España, en diversas oportunidades, durante los que fueron por su parte los difíciles años chilenos que corrieron hasta 1990. En uno de esos viajes conocí a Elías Díaz y desde ese mismo momento he mantenido con él un modo de relación que yo me atrevo a calificar con una hermosa palabra: amistad.

Durante casi veinte años, en consecuencia, he recibido de él, como muchos otros dentro y fuera de España, inspiración, guía y estímulo para perseverar en mi trabajo académico en el campo de la Filosofía del Derecho, y -lo que es ciertamente más importante-, para mantener en pie una preocupación a la vez teórica y práctica por los demás de la democracia y los derechos humanos.

En cuanto a «Doxa», cuyo décimo aniversario coincide con su número de homenaje a Elías Díaz, es para mí hace ya tiempo la mejor publicación periódica en lengua castellana en temas de Filosofía y Teoría del Derecho. Tuve el privilegio de colaborar con sus primer número y ese privilegio se repite ahora al cabo de un decenio. Espero simplemente que esta colaboración perdure por mucho tiempo más. 


\section{II \\ Sobre epígrafes}

Los dos epígrafes que hacen cabeza de este artículo han sido escogidos por las mismas e invariables razones que se tiene siempre para preferir una $u$ otra de estas sentencias: dicen bien y concisamente algo que a uno le habría gustado poder decir de la misma forma, y anticipan, resumiéndolas y confiriéndoles así mayor eficacia, algunas ideas centrales que se desearía transmitir como resultado de lo que se apresta a escribir.

La primera de tales citas está tomada de la que posiblemente sea la mejor de las novelas de la reputada autora inglesa de obras policiales. En cuanto a la segunda, pertenece al riguroso perspicaz filósofo chileno, a quien debemos en nuestro medio -amén de otras contribuciones de importancia- un espléndido ensayo sobre el tema de la violencia y de las máscaras que ésta adopta para aparecer revestida de buenas intenciones.

La cita de la novelista sugiere una visión algo naturalista de la violencia, esto es, su percepción como un simple y viejo dato que se halla presente, desde siempre, tanto en la naturaleza como en la sociedad. Millas no habría estado muy de acuerdo con este enfoque, porque pensaba que la historia no sólo tiene una dimensión fáctica, sino también ética, lo cual quiere decir que el hombre no presencia y expone únicamente la historia, sino que también, en alguna medida, la hace; y porque, además, en lo que a la vida humana concierne, a diferencia de lo que pasa con la vida simplemente animal, la violencia se da «en el contexto de una conciencia que es capaz de juzgarla y controlarla».

En cuanto a la sentencia del filósofo acerca de las desgracias de nuestro tiempo, ella insinúa que en otras épocas el hombre ha sido ciertamente más desdichado que en la hora presente, lo cual puede parecer un aserto trivial, pero que invita a que procuremos echar sobre el tema de nuestros pesares -y entre éstos sobre la violencia- una mirada en la que la inmediatez del primer plano pueda ser sustituida por la holgura de una visión más panorámica.

Y para concluir con estas explicaciones sobre epígrafes, los dos escogidos nos gustan también porque, hallándose muy lejos de aprobar la violencia de nuestros días, sitúan el asunto en una cierta perspectiva, en parte desdramatizándolo, y favorecen así no una indiferencia frente al mismo, sino una actitud más racional y alejada todo lo que sea posible de la tan desmesurada como frecuente hipocondría social con que el tema suele ser analizado y expuesto por algunos líderes de opinión pública. 


\section{III}

\section{Sobre palabras}

Acerca del tema de la violencia, y específicamente de la fuerza, sólo puedo escribir con alguna propiedad a partir de la única formación que reconozco: la que habilita para ocuparse del derecho con fines de estudio y de enseñanza (eludo deliberadamente el empleo de la palabra «jurista», que, al menos en mis oídos, suena siempre, y de modo inevitable, algo presuntuosa).

Soy consciente, sin embargo, de que la obligada adopción del punto de vista del hombre sólo familiarizado con el derecho y la teoría jurídica, estrecha bastante el marco de los análisis que seguirán a continuación en este artículo, en el que procuraré describir las diferencias y relaciones entre derecho y fuerza y el modo como el primero, precisamente para que no reine la segunda, monopoliza el uso de ésta al interior de la sociedad, aunque con un éxito sólo relativo, puesto que la fuerza no desaparece por obra del derecho.

Pero antes de pasar a la parte siguiente, conviene detenerse un instante en el uso que daremos aquí a la palabra «violencia».

Hannah Arendt, en un texto ya clásico sobre el tema, constata que cualquier observador de la historia y de la política debe «reconocer a la fuerza el enorme papel que ha desempeñado la violencia en los asuntos humanos», motivo por el cual -insiste la autoraresulta «difícil explicarse por qué la violencia ha merecido tan poca consideración especial».

A esa misma pensadora se debe uno de los pocos intentos sistemáticos por distinguir entre algunas palabras claves de las ciencias políticas -tales como «poder», «poderío», «fuerza», «autoridad» $\mathrm{y}$ «violencia»-, que si bien designan fenómenos distintos o no exactamente coincidentes, son utilizadas comúnmente, sin embargo, como expresiones equivalentes.

Por mi parte, no intentaré siquiera enunciar, ni tampoco analizar y menos aún profundizar, las distinciones propuestas por Hannah Arendt sobre el particular, puesto que semejante tarea sobrepasa los límites del presente trabajo.

Sólo avanzaré, de la mano del uso común de las palabras, pero también del «Diccionario de Política» de Bobbio, Matteucci y Pasquino, el sentido en que utilizaremos aquí las palabras «violencia»y «fuerza», quedándonos en definitiva con la segunda de tales palabras, aunque empleándola, claro, en una acepción equivalente a la de la primera.

En general, se entiende por «violencia» la acción de violentar, 
esto es, de obrar con fuerza de modo de vencer la resistencia de una persona. Por su parte, obrar con «fuerza» importa obligar a uno a que dé asenso a una cosa, o a que la haga. Es así, entonces, como la palabra «violencia» se equipara a la expresión "fuerza», destacándose además ese sentido «instrumental» que posee la violencia y que ha sido muy bien destacado también por Hannah Arendt.

Precisando todavía un poco más, podríamos decir que violencia significa dar muerte o infligir sufrimiento, esto es, privar a alguien de un bien (la vida, la libertad, la propiedad), procediendo para ello en forma intencional y forzada, o sea, queriendo que esa privación se produzca y valiéndose para ello de la fuerza física: una acepción posiblemente restringida de la palabra, que, a la vez que permite equipararla a la expresión «fuerza» (en el sentido físico del término), sirve también mejor que otros de sus posibles sentidos al planteamiento que haremos a continuación acerca de las relaciones entre derecho y fuerza.

Hablaremos en adelante, pues, de «derecho y fuerza», y no de «derecho y violencia», porque, además de lo ya dicho, resulta también el modo que se corresponde mejor con el uso más habitual de estas palabras en la literatura jurídica que se ha ocupado del tema.

En consecuencia, la noción con la que trabajaremos se conecta con la de poder político. Así, si se parte de una idea muy amplia de poder, como la de Robert Dahl, en el sentido de entender por éste «una relación de influencia entre actores» en la que cada uno de ellos «induce a los otros a que actúen de un modo en el que de otra forma no actuarían», pueden luego admitirse, con Bobbio, tres tipos principales de poder social, a saber, económico, ideológico y político, y entender luego el poder político como «el poder cuyo medio específico es la fuerza, o sea, aquel poder en que el instrumento decisivo para imponer la propia voluntad es la fuerza». De este modo, y siempre de la mano de Bobbio, «el poder político poco a poco se identifica con el ejercicio de la fuerza y se define como ese poder que para obtener los efectos deseados tiene el derecho de utilizar, aunque en última instancia, como extrema ratio, la fuerza. Sin embargo, el uso de la fuerza física, si bien condición necesaria para la definición del poder político, no es condición suficiente, puesto que al simple ejercicio de la fuerza tiene que sumarse la exclusividad del derecho al ejercicio de ésta en un territorio determinado. Y concluye Bobbio, por lo mismo, en que «si el uso de la fuerza es la condición necesaria del poder político, solamente el uso exclusivo de este poder es su condición suficiente». 


\section{IV}

\section{Sobre derecho, fuerza y algunos malentendidos}

Kelsen no es el único jurista que ha visto las cosas de esta manera, aunque sí sea probablemente el que se expresó al respecto con mayor crudeza:

«¿Qué característica común podrían tener el llamado Derecho de los antiguos babilonios y el Derecho vigente en Estados Unidos, o el orden social de una tribu africana bajo la autoridad de un jefe despótico con la constitución de la República Suiza? Sin embargo, hay un elemento común que justifica por entero esta terminología, es decir, que permite a la palabra «Derecho» expresar un concepto de gran significación social. Porque esta palabra denota precisamente la técnica concreta de un orden coercitivo que, pese a las enormes diferencias que existen entre el Derecho de los antiguos babilonios y el Derecho vigente en Estados Unidos, entre el Derecho de los ashantis del África occidental y el de los suizos en Europa, es, no obstante, esencialmente la misma para todos esos pueblos tan ampliamente heterogéneos en tiempo, espacio y cultura, a saber: técnica social consistente en obtener de los hombres el comportamiento social que se desee, por medio de la amenaza de una medida coercitiva aplicable en caso de conducta contraria».

Lo que la cita precedente quiere destacar es que el derecho no se caracteriza sólo por ser un orden normativo, esto es, por procurar conseguir determinados comportamientos que se consideran socialmente deseables -declarar y pagar un determinado impuesto, abstenerse de causar daño a la propiedad de otro, etc.- bajo la amenaza de ciertas sanciones -que pueden ir desde la privación de la vida (pena de muerte) hasta la simple privación de parte del patrimonio (una multa)-, sino sobre todo, porque respecto de sus sanciones existe la legítima posibilidad de aplicarlas aun contra la voluntad del infractor, y, todavía más, en uso de la fuerza socialmente organizada.

El derecho es un orden normativo, como lo es también la moral, pero su especificidad se encontraría, principalmente, en su carácter coercible, entendida la nota de la coercibilidad no como el hecho efectivo de la aplicación de la fuerza en presencia de cada infracción jurídica, sino, como se dijo, al modo tan solo de que, acaecida la infracción, se produce la legítima posibilidad de hacer aplicación de la fuerza para imponer al culpable la correspondiente sanción.

Dando cabida aquí a una distinción frecuente en el lenguaje de los juristas, la conexión entre derecho y fuerza, por lo mismo, no es externa, puesto que la fuerza no aparece como un simple medio para 
realizar el derecho; antes bien, la conexión sería de tipo interno, puesto que, tal como ha sido dicho, el derecho no se nos presenta como un conjunto de normas garantizadas por la fuerza, sino como un conjunto de normas que regulan el uso de la fuerza.

Claro: el derecho que conocieron los antiguos babilónicos ha de haber sido bien distinto del derecho que rige hoy en un estado de Norteamérica. Distintos modos y órganos de producción del derecho, diferentes materias de regulación, muy distintos contenidos de las normas de uno y otro, manifiesta diversidad de las finalidades históricas perseguidas con uno y otro ordenamiento. Pero en ambos casos, según quiere decirnos Kelsen, de lo que se trata es de inducir determinados comportamientos bajo la amenaza de un castigo que resulta posible y legítimo imponer en uso de la fuerza más o menos centralizada y, a la par, socialmente organizada.

Con todo, hay que evitar el malentendido que consiste en creer que el derecho impone por la fuerza (física) los comportamientos que demanda como debidos, puesto que la verdad es muy otra: lo que el derecho puede legítimamente imponer por medio de la fuerza son, sobre todo, las sanciones, esto es, las consecuencias desfavorables que sus propias normas prevén para una vez que el comportamiento debido no haya sido observado por quien debía hacerlo.

Pero lo que pasa, incluso hasta hoy, es que los autores que, como Kelsen, describen de este modo el fenómeno jurídico, han quedado expuestos a que se deje caer sobre ellos una suerte de mala prensa que los acusa de estar confundiendo el derecho con la fuerza. Este es un nuevo malentendido que también convendría disipar.

El derecho se configura efectivamente como una organización de la fuerza al interior de la sociedad, pero ello no lo vuelve comparable a cualquier organización de la fuerza, puesto que el derecho, en cuanto modo de organizar socialmente el uso de la fuerza, tiene algunas características que le son propias, a saber, que determina con precisión cuáles serán las hipótesis conductuales que, realizadas luego por alguien, constituirán el antecedente de una sanción; que determina, con similar precisión, el contenido específico que tendrá la sanción en cada caso; que exige que la verificación de la hipótesis conductual que ha sido descrita como antecedente de una sanción, así como la declaración de esta última, sean hechas por un órgano estatal independiente y con poder suficiente para hacer cumplir sus decisiones; que otorga al afectado, esto es, a quien realizare la conducta que el ordenamiento jurídico describe como antecedente de una sanción, la posibilidad de ser oído y de defenderse dentro de un proceso con garantías de imparcialidad que le permitan demostrar que, en su caso, no procede la aplicación de la sanción o que ésta debe reducirse a una de un tipo y medida determinados; y que, por 
último, aplica la sanción por medio del uso de la fuerza, si fuere necesario, aunque no de la que pudiera hacer uso directamente el ofendido o perjudicado por la infracción, sino a través de órganos centralizados del propio Estado.

Las órdenes que da el derecho, por lo mismo, no son comparables, como se ha dicho más de alguna vez, a las órdenes que da una banda de ladrones. La orden de entregar su dinero que da el asaltante a su víctima tiene sólo el sentido subjetivo de un deber para ésta última, en cuanto ella siente que debe actuar, mejor, que tiene que actuar de acuerdo a la orden recibida, si es que no quiere perder la vida o ser lesionada, aunque ninguna norma válida le impone una obligación semejante. Por la inversa, en el caso de una ley que ordena el pago de un impuesto, ésta tiene el sentido objetivo de un deber para el contribuyente, desde el momento que reconoce una obligación sólo en cuanto hay una norma jurídica válida que la establece.

En consecuencia, el derecho no es lo mismo que la fuerza, aunque, a la vez, se nos muestra como una determinada manera de organizar la fuerza al interior de la sociedad. Esta curiosa relación del derecho con la fuerza -no ser lo mismo que ésta y no poder existir sin ella-, queda muy bien puesta de manifiesto si se piensa en el modo como suele ser representado el derecho, esto es, por medio de la figura de una mujer que en una de sus manos sostiene una balanza, en tanto que en la otra blande una espada. La balanza es donde se pesa el derecho; la espada, por su parte, sirve para hacerlo cumplir. Un derecho sin la espada sería un derecho impotente, incapaz de imponer sus prescripciones; un derecho sin la balanza sería sólo la fuerza bruta, libre de todo control. Por eso es que -como advierte Ihering-, «el derecho no reina verdaderamente sino allí donde la fuerza que se emplea en blandir la espada iguala a la habilidad que se utiliza para sostener y manejar la balanza».

Por otro lado, a veces se pone en duda el carácter coactivo del derecho, diciendo para ello que no todas las normas de un derecho dado establecen directamente sanciones que es posible aplicar por medio de la fuerza. Esto es correcto hasta cierto punto. De hecho, en todo ordenamiento jurídico hay enunciados que no establecen sanciones e, incluso, que no establecen siquiera una conducta como debida. Tal ocurre, por ejemplo, con aquellos que permiten una conducta, o que facultan a alguien para producir nuevas normas, o que definen un concepto. Pero, según nos parece, nada de ello obsta a que el derecho, globalmente considerado, continúe siendo algo que tiene que ver preferentemente con normas $\mathrm{y}$, en especial, con normas coactivas.

Es en tal sentido, además, que puede afirmarse que el derecho se atribuye el monopolio del uso de la fuerza. «El desarrollo del derecho positivo -escribe otra vez Kelsenconduce forzosamente a que 
no esté llamado a la realización del acto coactivo -como ocurría primitivamente- el perjudicado en sus intereses, sino un «órgano» especial que funciona según el principio de la división del trabajo». Y en un sentido muy similar, Max Weber anota que «en la actualidad, debemos decir que un Estado es una comunidad humana que se atribuye (con éxito) el monopolio del uso legítimo de la fuerza dentro de un territorio dado».

Ahora bien, si el derecho se atribuye el monopolio de la fuerza, no es ciertamente para reducirse a ésta y legitimar su reinado entre los hombres. Todo lo contrario, el derecho procede de ese modo con el fin de conseguir la paz, esto es, de eliminar toda otra fuerza que no sea la propia. Si el derecho no se atribuyera el monopolio del uso de la fuerza, centralizando su ejercicio a través de órganos jurisdiccionales y coactivos independientes, entonces sí que lo más probable sería que el devenir de las relaciones sociales quedara librado a lo que usualmente se llama «la ley del más fuerte».

Claro que en todo ello el derecho tiene sólo un éxito relativo. Relativo, en primer lugar, porque al atribuirse el monopolio del uso de la fuerza, no elimina del todo a ésta, sino únicamente la centraliza, desde el momento que el propio derecho reaccionará a su turno con actos de fuerza, aunque legítimos, en presencia de conductas que él haya descrito como no debidas. Y relativo, en segundo término, porque si bien el derecho procura inducir comportamientos por medio de la amenaza de sanciones coactivas, lo cierto es que este último tipo de actos continuará produciéndose en alguna medida, movilizando así el aparato coercitivo dispuesto por el propio derecho y mostrando que éste es siempre sólo parcialmente eficaz en el obedecimiento que consigue suscitar, de hecho, a las normas que ha prescrito como debidas, aunque cuenta siempre en tales casos con la posibilidad de resultar eficaz en cuanto a la aplicación de las sanciones que haya previsto, precisamente, para los casos de desobedecimiento a tales normas por parte de los correspondientes sujetos imperados.

Una descripción semejante del derecho, y de sus relaciones y diferencias con la fuerza, que muestra, según hemos visto, que aquél no se reduce a ésta pero tampoco le resulta posible existir sin ella, puede promover la sensación de que estamos ante toda una paradoja. Pero ello, de ser así, no debería asustarnos. Las paradojas son siempre mejores que los prejuicios, según decía Rousseau. Y si el derecho aparece en un punto demasiado cercano a la fuerza, aunque a la vez justo a tiempo para diferenciarse de ésta, amén de la anunciada paradoja, ello puede constituir una advertencia, o al menos un simple indicio, de que los hombres no hemos podido aún producir otro instrumento mejor y más eficaz para controlar el uso de la fuerza y evitar que ésta se enseñoree en el campo de las relaciones interpersonales 
y sociales, sin perjuicio de que lo que derecho logra o procura conseguir a este respecto, si bien imperfecto, no es poca cosa: si no es posible suprimir del todo la fuerza, al menos el monopolio de su uso por parte del derecho, en los términos y condiciones que fueron mencionados antes, garantiza una cierta paz, una paz relativa, en el curso de la vida social.

Por último, la visión que hemos ofrecido aquí del derecho puede parecer algo simple, y la verdad que lo es. El derecho, por cierto, no cumple sólo una función coactiva y a posteriori, sino también funciones promocionales (cuando incentiva determinados comportamientos mediante recompensas o beneficios) y distributivas (reparto de bienes y de oportunidades).

Además, si nos quedamos sólo con la función coactiva del derecho, podemos comprobar que esta función ha ido en cierta medida debilitándose en la sociedad actual, desde el momento -primero- que otras técnicas distintas del derecho, tales como la publicidad y los medios de comunicación, se están mostrando cada vez más eficaces como instrumentos dirigidos al incentivar comportamientos, y establecido -segundo- que la sociedad moderna, a través de ciencias como la Sociología y la Psicología, como nos recuerda Manuel Atienza, se orienta antes a prevenir que a sancionar comportamientos no deseados.

Pero en este trabajo nuestra perspectiva ha sido tan sólo la de quien procura describir el carácter coactivo del derecho. Asumir el fenómeno jurídico en toda su complejidad constituye ciertamente una exigencia, pero ese no era el propósito de estas líneas.

\section{BIBLIOGRAFÍA}

Hannah Arendt, «Sobre la violencia», Cuadernos Joaquín Moritz, México, 1970, traducción de Miguel González.

Manuel Atienza, «Introducción al Derecho», Barcanova, Barcelona, 1985.

Norberto Bobbio, Nicola Mateucci y Gianfranco Pasquino, «Diccionario de Política», Siglo XXI Editores, México, 1981, traducción de Raúl Crisaglio, Alfonso García, Mariano Martín y Jorge Tula. Redactores de la edición en español, José Aricó y Jorge Tula.

Robert Dahl, «Modern political analysis», Prentice Hall, Englewood Cliffs, 1963.

Rudolf von Thering, «La lucha por el derecho», en Revista de Ciencias Sociales n ${ }^{\text {os }}$. 10-11, 1979, Facultad de Derecho y Ciencias Sociales de la Universidad de Valparaíso, Edeval, Valparaíso.

P. D. James, «Intrigas y deseos», Javier Vergara Editor, Buenos Aires, 1990, traducción de César Aira.

Hans Kelsen, «Teoría Pura del Derecho», UNAM, México, 1979, traducción de Roberto J. Vernengo; «Derecho y Paz en las relaciones internacionales», 
Fondo de Cultura Económica, México, 1986, traducción de Francisco Acosta.

Jorge Millas, «Las máscaras filosóficas de la violencia», en La violencia y sus máscaras, Editorial Aconcagua, Santiago, 1978.

— Opiniones de Jorge Millas», en Anuario de Filosofía Jurídica y Social no . 2, «Estudios en memoria de Jorge Millas», 1984, Sociedad Chilena de Filosofía Jurídica y Social, Valparaíso.

Max Weber, «La política como vocación», en Ensayos de sociología contemporánea, Ediciones Martínez-Roca, Barcelona, 1972, traducción de Mireia Bofill. 